J. Phys. IV France 138 (2006) 191-201

(C) EDP Sciences, Les Ulis

DOI: $10.1051 / \mathrm{jp} 4: 2006138022$

\title{
Mécanismes d'éjection de particules par laser impulsionnel
}

\author{
D. Grojo ${ }^{1}$, A. Cros $^{1}$, Ph. Delaporte ${ }^{1}$, M. Sentis ${ }^{1}$, H. Dubus ${ }^{2}$ et R. Mionetto ${ }^{2}$ \\ ${ }^{1}$ LP3 - UMR 6182 CNRS, Université de la Méditerranée, Campus de Luminy, \\ Case 917, 13288 Marseille Cedex 9, France \\ 2 STMicroelectronics Rousset, 13106 Rousset, France
}

\begin{abstract}
Résumé. L'enlèvement de particules de dimensions nanométriques est l'un des principaux challenges à relever pour atteindre les futurs objectifs de l'industrie microélectronique. Les procédés laser présentent, dans certains cas, des performances très intéressantes, mais les mécanismes d'éjection des particules polluant la surface restent cependant fort mal connus. L'étude de la dynamique d'éjection des particules, par une technique optique, a mis en évidence l'existence de deux mécanismes dont l'importance relative dépend de la fluence d'irradiation. A forte fluence l'ablation locale du substrat sous la particule prédomine, alors que pour les fluences plus faibles le mécanisme semble être lié à l'enlèvement de l'humidité résiduelle à l'interface particule - substrat. Contrairement aux modèles précédemment proposés, la contribution de la force d'inertie s'exerçant sur la particule lors de l'expansion thermique rapide des matériaux est négligeable.
\end{abstract}

\section{INTRODUCTION}

Le développement des nanotechnologies implique l'utilisation et la création d'objets de tailles nanométriques. Cela signifie, en autres, qu'il est nécessaire de tenir compte des polluants dont les dimensions sont dans ces ordres de grandeur et qui jusqu'à présent pouvaient être négligés. L'enlèvement de ces nanoparticules représente donc un enjeu stratégique dans des domaines tels que la microélectronique, l'optique ou l'optoélectronique. De plus, le développement de procédés de nettoyage particulaire sans contact est également important pour la décontamination en milieu nucléaire ou bactériologique. Dans l'industrie microélectronique, l'augmentation continuelle de la densité d'intégration des composants entraîne la réduction de la taille des défauts acceptables alors que les surfaces deviennent de plus en plus fragiles. Les techniques chimiques actuellement utilisées induisent une érosion de la surface incompatible avec les épaisseurs de plus en plus fines des prochaines générations de composants, et les procédés utilisant les ultrasons présentent des risques d'endommagement trop grands pour les structures de la surface. Dans ce contexte, l'enlèvement de nanoparticules de la surface des wafers de silicium nécessite le développement de nouveaux procédés. Trois techniques basées sur l'utilisation de lasers impulsionnels ont été proposées pour relever ce déf : Le 'Dry Laser Cleaning' (DLC) [1, 2] pour laquelle le faisceau irradie directement la surface, le 'Steam Laser Cleaning' (SLC) $[3,4]$ qui nécessite le dépôt d'un film liquide très fin avant l'irradiation, et le 'Laser induced Shock wave Cleaning' (LSC) [5] qui utilise les ondes de chocs générées par un claquage laser de l'air juste au-dessus de la surface pour éjecter les particules. Les travaux présentés dans cet article concernent le premier de ces procédés qui présente l'avantage d'être sec, d'avoir des performances prometteuses et dont la mise en œuvre industrielle est aisée.

La figure 1 illustre l'efficacité potentielle de cette technique. L'enlèvement de particules de polystyrène de $190 \mathrm{~nm}$ de rayon, déposées sur une surface de silicium, est réalisé en un seul tir laser et pour des fluences de quelques centaines de $\mathrm{mJ} / \mathrm{cm}^{2}$. Au cours de ces dernières années, ce procédé a fait l'objet de nombreuses études expérimentales [6,7] et plusieurs descriptions théoriques ont été proposées $[8,9]$. Ces dernières suggèrent différents mécanismes d'éjection tels que l'expansion rapide du substrat due à son échauffement par le faisceau [10], l'ablation locale du substrat sous la particule induite par une exaltation du champ par celle-ci [11], ou encore l'évaporation explosive de l'humidité résiduelle se trouvant à l'interface particule-substrat [12]. La plupart des modèles numériques développés pour 
simuler ce processus d'enlèvement sont basés sur l'effet mécanique induit par l'expansion d'origine thermique de la surface. Bien que certains d'entres eux prennent en compte des effets tels que la surintensité locale d'énergie sous la particule ou sa réponse élastique [10], ils ne permettent pas de décrire de manière satisfaisante les résultats expérimentaux, surtout d'un point de vue quantitatif. Afin de d'identifier les mécanismes responsables de l'éjection des particules induite par l'irradiation laser, de nombreuses études expérimentales ont été réalisées. Elles concernent notamment l'influence de la taille des particules, de la nature du couple substrat - particule et des paramètres d'irradiation sur l'efficacité du procédé [13], la caractérisation du mouvement de la surface due à l'irradiation [14]. Il n'y a cependant, à ce jour, aucune démonstration expérimentale du processus prédominant d'éjection des particules. Ces travaux ont donc pour objet d'étudier la dynamique d'éjection des particules en utilisant une technique optique basée sur la diffusion de ces dernières lors de leur traversée d'un faisceau sonde. Ils ont pour but de déterminer les mécanismes responsables de l'enlèvement des particules. Des résultats d'enlèvement de résidus polymères polluant des wafers sont également présentés.

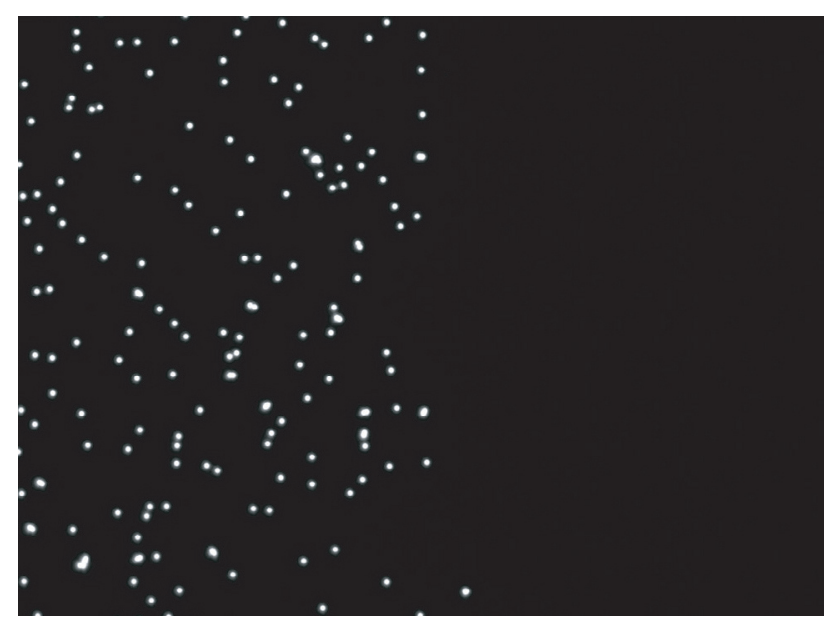

Figure 1. Enlèvement de particules de polystyrène de $380 \mathrm{~nm}$ de diamètre par irradiation avec un laser $\mathrm{XeCl}$. La partie droite du substrat de silicium a été irradiée avec 10 tirs laser à $450 \mathrm{~mJ} / \mathrm{cm}^{2}$.

\section{DISPOSITIF EXPÉRIMENTAL}

Les expériences de nettoyage de surface ont été réalisées avec un laser à excimères $\operatorname{ArF}(\lambda=193 \mathrm{~nm}$, Lambda Physik LPX220i) dont les impulsions ont une durée à mi-hauteur de 15ns et une énergie maximale de $300 \mathrm{~mJ}$. Les substrats sont irradiés par une seule impulsion laser. Un système optique simple permet d'obtenir une répartition quasi-uniforme de l'énergie du faisceau sur la surface. Il comprend un masque métallique à ouverture rectangulaire réglable dont l'image est faite dans le plan du substrat au moyen d'une lentille de focale $250 \mathrm{~mm}$. La zone irradiée a une surface de 2 x 2,6 $\mathrm{mm}^{2}$. L'énergie de l'impulsion laser peut être modifiée manuellement de manière continue à l'aide d'un atténuateur (Optec, AT4030).

Les surfaces traitées sont des substrats de silicium (1.0.0.) provenant de wafers commerciaux de dimensions 10 x $10 \mathrm{~mm}^{2}$. Les particules sont des sphères calibrées commerciales (Kisker-Biotech) en silice de rayon 250nm. La solution collö̈dale initiale est diluée dans de l'isopropanol (IPA). Les substrats sont d'abord nettoyés dans un bain à ultrasons, puis les particules sont déposées sur la surface par une 
technique de 'spin-coating'. Ce procédé permet d'obtenir sur la surface des densités de particules de l'ordre de $10^{9}$ particules $/ \mathrm{m}^{2}$ avec plus de $90 \%$ de particules isolées.

Un microscope optique (Olympus) équipé d'une caméra numérique (Qimaging) est utilisé pour visualiser les échantillons in situ. Un logiciel de détection et de comptage de particules nous permet de calculer l'efficacité du nettoyage entre chaque irradiation laser. Les visualisations plus précises des particules sur la surface et des endommagements éventuels de cette dernière sont réalisées avec un microscope à balayage électronique (M.E.B.) JEOL 6320F à émission de champ.

La figure 2 présente un schéma de principe (2.a) et une photographie (2.b) du dispositif utilisé pour réaliser les mesures de temps de vol des particules éjectées. La détection individuelle de particules de taille nanométrique ne pouvant être effectuée par un dispositif conventionnel d'observation à longue distance, nous avons utilisé une approche expérimentale basée sur la diffusion pour détecter la présence d'un ensemble de particules. Pour éviter le redépôt des particules sur la surface et une réduction trop importante de la vitesse des particules éjectées par collisions avec les atomes du gaz environnant, les substrats sont placés à l'intérieur d'une cellule dans laquelle une pression résiduelle de $5.10^{-2} \mathrm{~Pa}$ est maintenue. Lors de l'irradiation de la surface, l'éjection des particules se fait perpendiculairement à la surface. Ces dernières, en traversant un faisceau laser sonde qui se propage parallèlement au substrat, diffusent une partie de la lumière de ce faisceau, permettant ainsi leur détection collective. La cellule est équipée de trois fenêtres optiques. La première permet le passage du faisceau du laser ArF, le faisceau sonde pénètre dans la cellule par la seconde et la dernière est utilisée pour visualiser la lumière diffusée par les particules. Cette détection est réalisée à l'aide d'une caméra CCD intensifiée (Princeton Instruments, 576/RB-E) et d'un dispositif optique conçu autour d'un objectif photographique (Pentax FAJ 75-300mm). L'acquisition des images est synchronisée par rapport au déclenchement du laser. La résolution spatiale de cet ensemble est de $4 \mu \mathrm{m}$. Le laser sonde est un Nd :YAG continu émettant à $532 \mathrm{~nm}$ avec une puissance de $20 \mathrm{~mW}$ et un diamètre à $1 / \mathrm{e}^{2}$ de $0.9 \mathrm{~mm}$. Le profil d'énergie du faisceau au niveau de la surface, réalisé à l'aide d'une lentille cylindrique de focale $50 \mathrm{~mm}$, est une fine nappe de lumière affleurant la surface et coupant l'axe Z (cf. fig. 2.a) avec un angle de $20^{\circ}$. La détection de la lumière diffusée se fait donc le long de l'axe Z. Pour garantir une réponse linéaire de l'intensité lumineuse détectée par rapport à la densité de particules présentes dans le faisceau sonde, nous nous sommes assurés que la répartition d'énergie de ce faisceau le long de l'axe Z et sur la longueur d'analyse était uniforme.

(a)

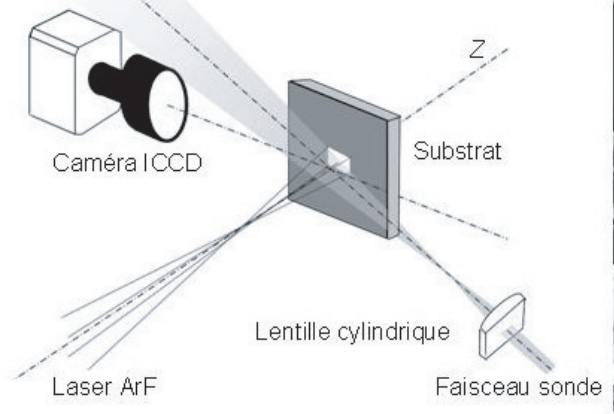

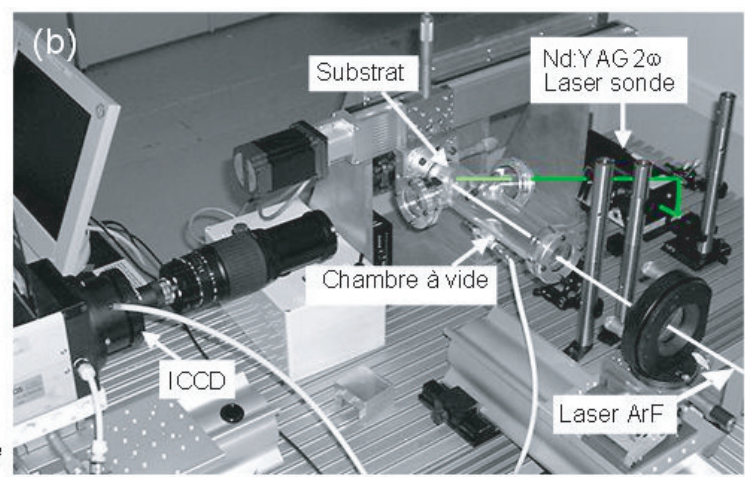

Figure 2. Schéma (a) et photographie (b) du dispositif expérimental utilisé pour détecter le nuage de particules par diffusion. Le faisceau du laser sonde (cw Nd :YAG @ 532nm) fait un angle de $20^{\circ}$ par rapport à la direction d'observation. La cellule permettant de maintenir sous l'échantillon sous une faible pression résiduelle est visible sur la photographie (b). 


\section{PRINCIPE DES MESURES DE TEMPS DE VOL}

Le principe de la diffusion de la lumière par des particules sphériques est largement utilisé pour développer des moyens de diagnostics. Une étude théorique [15], basée sur la théorie de Mie, nous a permis de définir les différents éléments de notre dispositif expérimental. On constate notamment sur figure 3 que pour détecter des particules de 250nm de rayon, la polarisation du faisceau sonde ne joue pas un grand rôle mais que l'intensité de la lumière diffusée dépend fortement de la direction d'observation. Dans le cas présent, il est important de placer la caméra CCD selon une direction proche de l'axe de propagation du faisceau sonde.

Lorsque la fluence d'irradiation laser est supérieure à la fluence seuil de nettoyage $\left(\mathrm{F}_{\text {th }} \approx 130 \mathrm{~mJ} / \mathrm{cm}^{2}\right)$ les particules sont éjectées de la surface, traversent le faisceau sonde, et diffusent de la lumière qui est enregistrée par la caméra CCD. La figure 4 représente une série caractéristique d'images du nuage de particules enregistrées avec différents délais temporels entre l'irradiation laser et l'instant d'acquisition. Les délais varient entre 10 et $120 \mu$ s et la porte d'observation est de $1 \mu \mathrm{s}$. A partir de ces images, il est possible de déterminer le profil de l'intensité de la lumière en fonction de la distance z entre la surface et la zone d'observation. La figure 5 présente trois profils d'intensité enregistrés pour trois délais différents. Chacun de ces profils peut être approximé par une fonction maxwellienne [15].
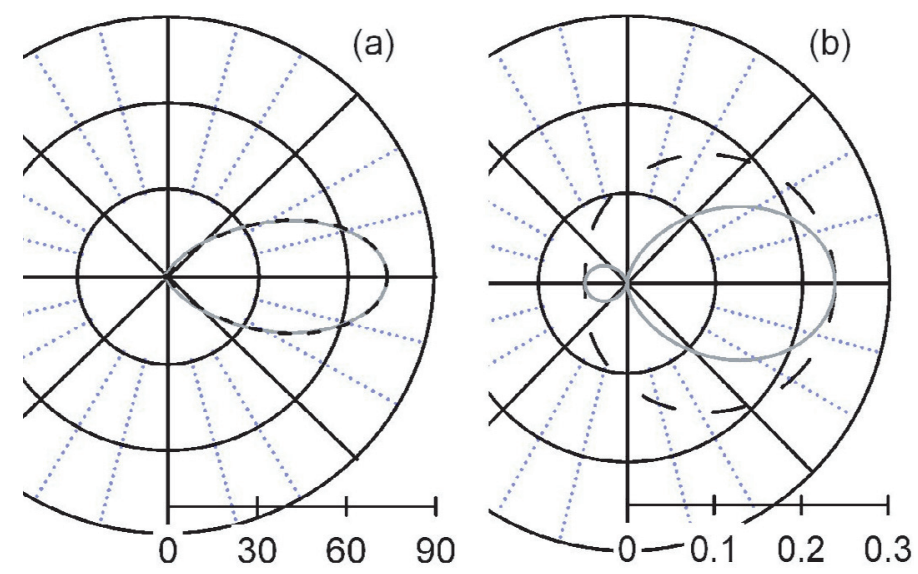

Figure 3. Diagrammes polaires pour la diffusion de lumière polarisée linéairement $(\lambda=532 \mathrm{~nm})$ par une sphère transparente d'indice de réfraction $\mathrm{n}=1,5$ et de rayon $250 \mathrm{~nm}$ (a) et $100 \mathrm{~nm}$ (b). Les lignes continues grises (resp. en pointillées noires) correspondent à une polarisation parallèle (resp. perpendiculaire).

Le graphe de la figure 6 montre la position du maximum d'intensité en fonction du délai pour deux fluences d'irradiation. On constate que la distance entre le maximum d'intensité et la surface varie linéairement avec le délai, ce qui signifie que la vitesse du nuage est constante, même pour une fluence proche de la fluence seuil de nettoyage, et donc que les interactions entre les particules et la pression résiduelle du gaz dans la cellule expérimentale sont négligeables dans la zone d'analyse. Dans le cas présent, les vitesses d'éjection mesurées sont de $9 \mathrm{~ms}^{-1}$ à $165 \mathrm{~mJ} / \mathrm{cm}^{2}$ et de $20 \mathrm{~ms}^{-1}$ à $270 \mathrm{~mJ} / \mathrm{cm}^{2}$.

\section{MÉCANISMES D’ÉJECTION}

\subsection{Résultats}

La figure 7 présente les images du nuage de particules éjectées, capturées pour différents délais et pour trois fluences d'irradiation. Elles montrent clairement que la vitesse des particules augmente avec la fluence. De plus, on peut observer sur les images correspondant à la fluence de $410 \mathrm{~mJ} / \mathrm{cm}^{2}$ la présence de 


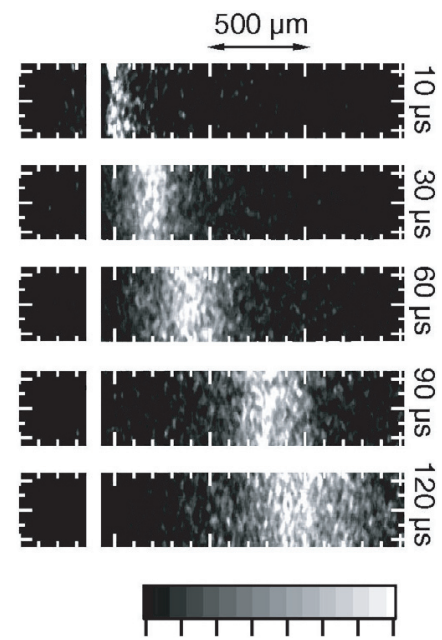

Figure 4. Images du nuage de particules diffusant la lumière du laser sonde durant l'éjection des particules pour différents délais entre le tir laser et l'acquisition. La fluence laser était de $165 \mathrm{~mJ} / \mathrm{cm}^{2}$ et le temps d'acquisition de $1 \mu \mathrm{s}$. Pour chaque image, le trait blanc représente la position du substrat et le laser ArF arrive du côté droit de la figure.

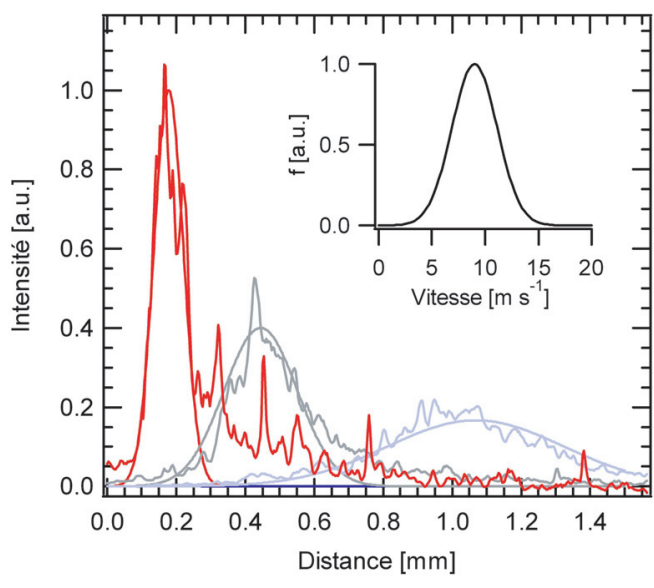

Figure 5. Intensité de la lumière diffusée en fonction de la distance avec la surface. Les courbes sont tracées à partir de l'analyse d'images similaires à celles de la figure 4 , pour des délais de 20,50 et $120 \mu$ s et une fluence de $165 \mathrm{~mJ} / \mathrm{cm}^{2}$. Les courbes d'intensité lissées sont calculées en supposant une distribution des vitesses décrites par la courbe insérée dans le graphe.

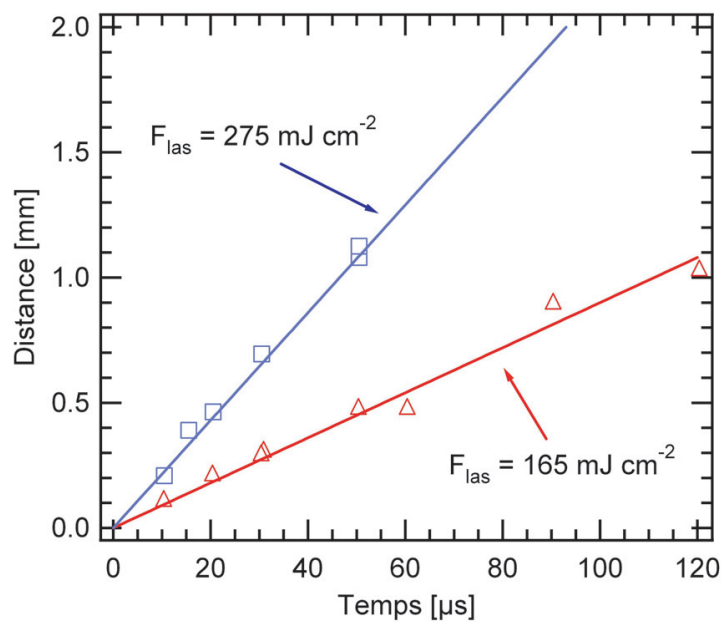

Figure 6. Distance entre le maximum d'intensité et la surface en fonction du délai entre l'acquisition et le tir laser pour deux fluences d'irradiation laser $\left(165 \mathrm{~mJ} / \mathrm{cm}^{2}\right.$ et $\left.275 \mathrm{~mJ} / \mathrm{cm}^{2}\right)$.

deux nuages de particules distincts. Ceci pourrait signifier qu'il y aurait deux mécanismes responsables de l'éjection des particules.

Les différentes mesures de vitesse d'éjection réalisées en fonction de la fluence d'irradiation sont représentées sur le graphe de la figure 8. L'efficacité du nettoyage est également indiquée sur la partie supérieure de cette figure. Ces résultats mettent clairement en évidence l'existence de deux régimes d'enlèvement des particules et donc de deux mécanismes distincts. De la fluence seuil jusqu'à 

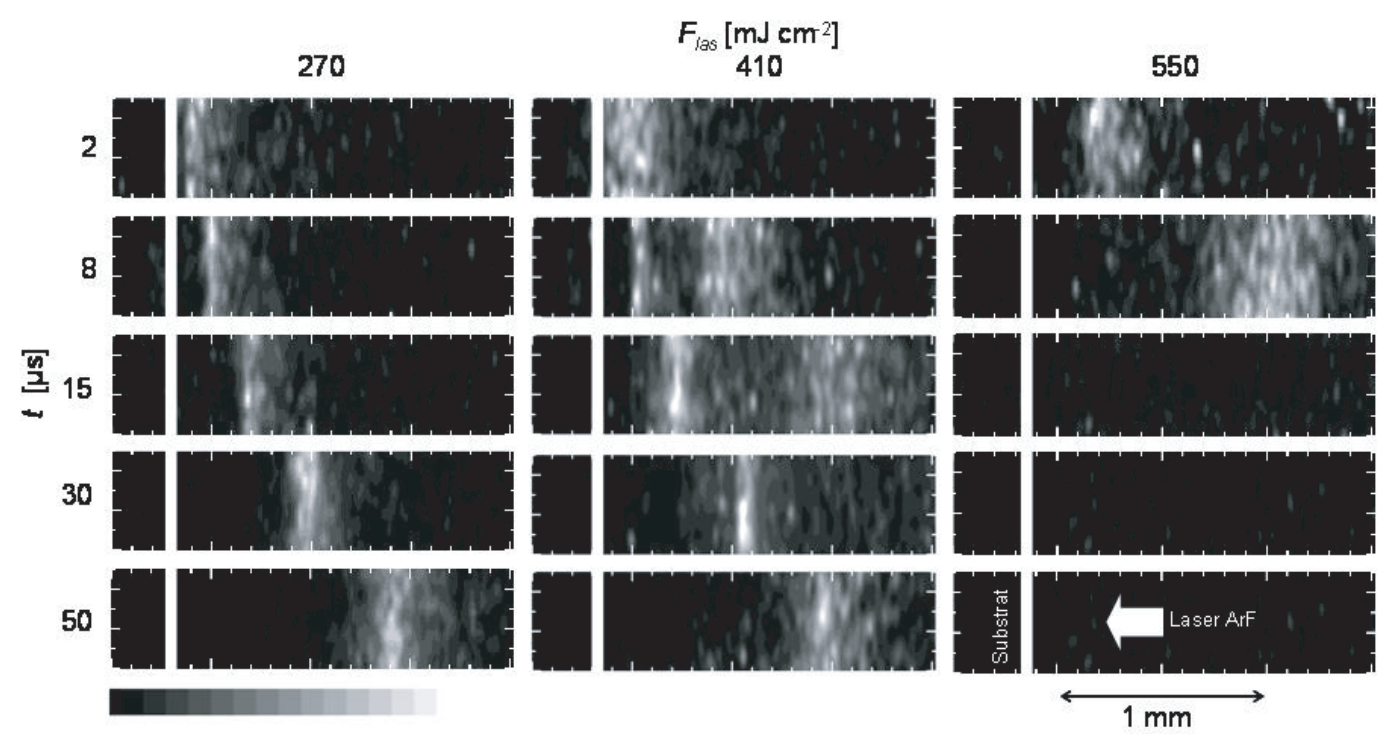

Figure 7. Images du nuage de particules diffusant la lumière du laser sonde durant l'éjection des particules pour différents délais entre le tir laser et l'acquisition, et pour trois fluences d'irradiation $\left(270,410 \mathrm{et} 550 \mathrm{~mJ} / \mathrm{cm}^{2}\right)$. Le temps d'acquisition est de $1 \mu$ s, et pour chaque image, le trait blanc représente la position du substrat.

$\mathrm{F}=500 \mathrm{~mJ} / \mathrm{cm}^{2}$, on mesure des vitesses relativement faibles qui varient linéairement avec la fluence de $7,6 \mathrm{~ms}^{-1}$ à $36,8 \mathrm{~ms}^{-1}$. Pour les fluences plus élevées, la variation de la vitesse en fonction de la fluence est toujours linéaire, mais beaucoup plus rapide. Elle passe de $66 \mathrm{~ms}^{-1}$ pour $\mathrm{F}=415 \mathrm{~mJ} / \mathrm{cm}^{2}$ à $231 \mathrm{~ms}^{-1}$ pour $\mathrm{F}=680 \mathrm{~mJ} / \mathrm{cm}^{2}$. Lors des expériences menées dans des conditions d'irradiation pour lesquelles les deux nuages sont observables simultanément $\left(300 \mathrm{~mJ} / \mathrm{cm}^{2}<\mathrm{F}<500 \mathrm{~mJ} / \mathrm{cm}^{2}\right)$, on a observé qu'une augmentation progressive de la fluence induisait une diminution de l'intensité de la composante la plus lente alors que celle de la composante rapide augmentait. Ce comportement montre bien que le processus de nettoyage résulte du rôle de deux mécanismes dont l'importance relative dépend de la fluence.

\subsection{Discussion}

Depuis les premières démonstrations expérimentales de l'efficacité du processus d'enlèvement de particules par laser (DLC), de nombreux modèles théoriques ont été développés. Ils attribuent, pour la plupart, l'éjection des particules à un effet mécanique lié à l'expansion rapide de la surface due à son échauffement induit par l'irradiation laser [8-10]. Les plus simples se contentent de résoudre l'équation de la chaleur avec une approche unidimensionnelle pour calculer le profil de température $\mathrm{T}(\mathrm{z}, \mathrm{t})$, et d'en déduire, en résolvant l'équation de thermoélasticité, le déplacement de la surface.

$$
z(t)=\alpha_{s} \int_{-\infty}^{0} T(z, t) d z
$$

Où $\alpha_{\mathrm{s}}$ représente le coefficient linéaire d'expansion thermique du matériel. Dans le cas monodimensionnel, la résolution de l'équation de la chaleur peut être évitée en considérant l'équation 


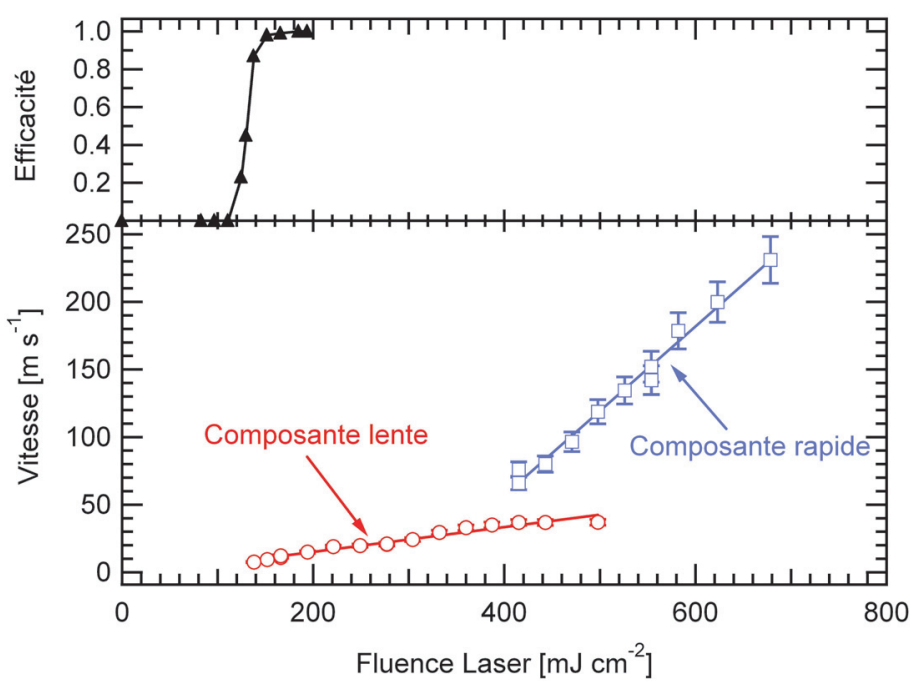

Figure 8. Efficacité du processus d'enlèvement de particules et vitesses des nuages de particules mesurées par diffusion en fonction de la fluence d'irradiation laser.

de conservation de l'énergie.

$$
(1-R) \int_{0}^{t} I\left(t^{\prime}\right) d t^{\prime}=\rho C_{p} \int_{-\infty}^{0} T(z, t) d z
$$

Où $\mathrm{C}_{\mathrm{p}}$ et $\rho$ sont la capacité calorifique et la densité spécifique du substrat, dans le cas présent du silicium. $\mathrm{R}$ est le coefficient de réflectivité de la surface et I l'intensité laser incidente. A partir de ces deux équations, on peut exprimer la vitesse $\mathrm{u}_{z}(\mathrm{t}) \mathrm{de}$ la surface.

$$
u_{z}(t)=\frac{\alpha_{s}}{\rho C_{p}}(1-R) I(t)
$$

Afin de calculer le déplacement la surface à proximité des particules, nous avons développé un modèle thermoélastique plus sophistiqué [7]. Il tient compte de l'augmentation locale de l'intensité laser sous la particule due à un effet d'exaltation en champ proche. Cet effet est décrit par la théorie de Mie, et l'équation de la chaleur est ensuite résolue en trois dimensions dans un système à symétrie cylindrique.

$$
\rho C_{p} \frac{\partial T}{\partial t}=G(z, r, t)+K_{t h} \Delta T(z, r, t)
$$

$\mathrm{K}_{\text {th }}$ est la conductivité thermique du substrat.

Les résultats obtenus avec ces modèles $1 \mathrm{D}$ et 3D sont présentés sur la figure 9. La simulation, qui tient compte de la surintensité induite sous la particule prédit des vitesses d'éjection légèrement supérieures à celles prédites par le modèle unidimensionnel. Les valeurs maximales des vitesses atteintes par la surface restent cependant de l'ordre du $\mathrm{cms}^{-1}$. Ces valeurs sont en parfait accord avec des mesures expérimentales effectuées par Dobler [14]. On constate donc que la vitesse de la surface est toujours inférieure, d'au moins deux ordres de grandeur, à la vitesse des particules que nous avons mesurées lors de ces expériences (figure 8). Si l'on considère le phénomène de déformation élastique de la particule qui peut contribuer de manière significative à son éjection $[10,16]$, les calculs montrent que ce processus ne devient significatif que pour des durées d'impulsions très courtes $(\approx 3 \mathrm{~ns})$ ou des tailles de particules 


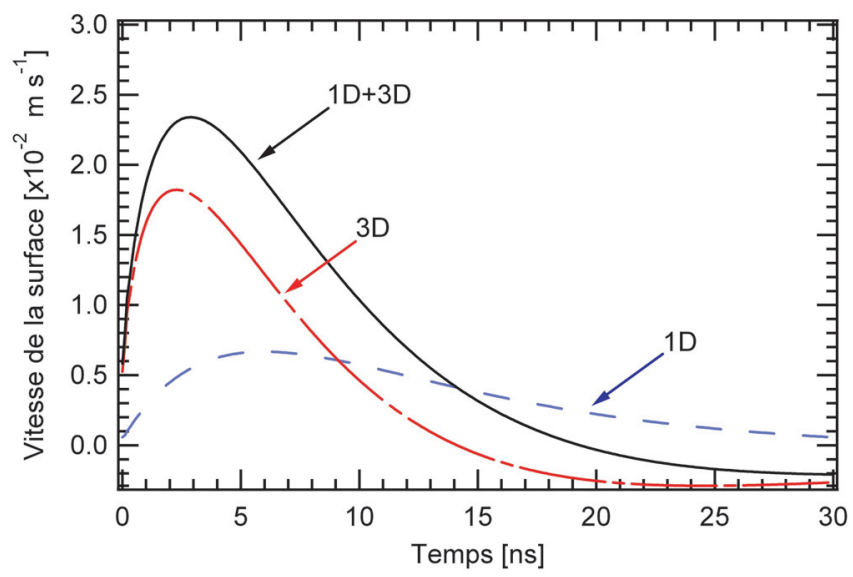

Figure 9. Vitesse d'expansion de la surface du substrat de silicium en fonction du temps. Les calculs sont effectués pour un profil temporel d'intensité de la forme $\mathrm{I}(\mathrm{t})=\mathrm{I}_{0}(t / \tau) \exp (t / \tau)$ avec une durée d'impulsion $\tau=15 \mathrm{~ns}$ et un profil spatial gaussien á la longueur d'onde $1 \lambda=193 \mathrm{~nm}$.

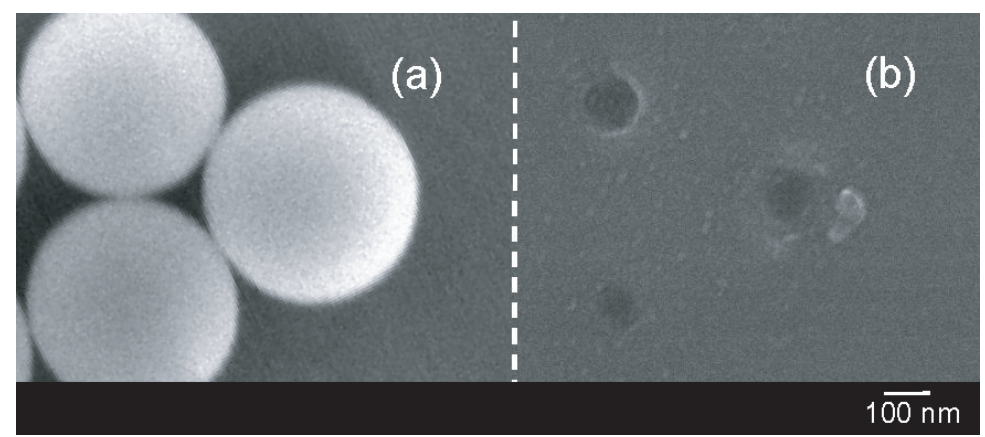

Figure 10. Images MEB de la surface avant (a) et après (b) l'irradiation laser à une fluence de $430 \mathrm{~mJ} / \mathrm{cm}^{2}$. L'image (b) montre les cratères dus à l'ablation du substrat sous la particule.

importantes. Il est donc possible d'affirmer que l'expansion rapide de la surface n'est pas un mécanisme responsable de l'éjection des particules, et donc du processus de nettoyage.

Afin d'identifier les mécanismes qui induisent l'enlèvement des particules, nous avons examiné les substrats par microscopie électronique après leur nettoyage par procédé laser. Comme le montre la figure 10, les substrats irradiés avec des fluences élevées, supérieures à $350 \mathrm{~mJ} / \mathrm{cm}^{2}$, présentent des cratères d'environ $100 \mathrm{~nm}$ de diamètre, situés à l'emplacement initial des particules. Cette ablation locale du substrat est due à la surintensité induite sous les particules par l'exaltation en champ proche. Ce phénomène correspond au régime pour lequel on observe une composante rapide du nuage de particules, et les vitesses d'éjection sont compatibles avec les vitesses habituellement observées lors des études sur l'ablation laser. Il est donc possible de conclure que le mécanisme responsable de l'enlèvement de particules en régime de fluence élevée est l'ablation locale du substrat. Il est clair que cet endommagement du substrat est inacceptable pour un procédé de nettoyage dans le domaine de la microélectronique, mais c'est une voie très prometteuse pour réaliser de la nanostructuration de surface [17].

L'identification du mécanisme à l'origine de l'éjection lors de l'irradiation à des fluences proches de la fluence seuil est plus complexe. Le processus qui, à ce jour, semble le plus probable est l'évaporation explosive de l'humidité résiduelle piégée entre la particule et le substrat [12,18]. Les causes de l'éjection 


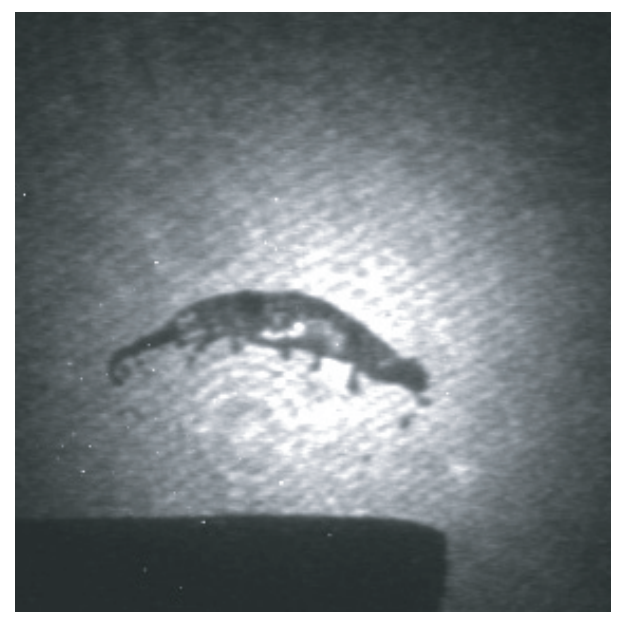

Figure 11. Ejection d'une goutte de liquide déposée sur une surface d'aluminium par irradiation laser à $248 \mathrm{~nm}$ et pour une fluence de $400 \mathrm{~mJ} / \mathrm{cm}^{2}$. Le mécanisme est l'évaporation explosive du liquide à l'interface surface-goutte. L'image a été acquise par une technique d'ombroscopie avec une caméra ICCD, $400 \mu$ s après le tir laser et pour un temps d'observation de 40ns.

seraient alors très similaires à ce qui est observée lors du nettoyage par procédé humide (SLC) qui consiste à enlever par irradiation laser un film liquide qui entraîne avec lui les particules [19, 20]. Comme le montre la figure 11, l'interaction se produit à l'interface liquide-substrat et le film est éjecté sans être détruit. Les vitesses d'éjection du film que nous avons mesurées et qui sont compatibles avec des expériences menées dans d'autres laboratoires sont comprises entre 10 et $40 \mathrm{~ms}^{-1}$ [21]. Ces vitesses sont du même ordre de grandeur que celles du nuage de particules mesurées lors de l'irradiation à faible fluence et qui correspondent à la composante lente de la figure 8 .

Des essais de nettoyage ont été réalisés en fonction du temps durant lequel l'échantillon restait dans l'enceinte dans laquelle un système de pompage maintenait une pression résiduelle de $5.10^{-2} \mathrm{~Pa}$. L'objectif était de réduire l'humidité en augmentant le temps de pompage. Notre dispositif ne nous a vraisemblablement pas permis d'enlever complètement cette humidité et donc de faire disparaître la composante lente du nuage de particules éjectées. Par contre nous avons constaté que le seuil d'enlèvement des particules diminuait de manière significative lorsque le temps de pompage augmentait, ce qui faisait baisser le taux d'humidité résiduelle. Ce résultat met en évidence que cette humidité contribue aux forces d'adhésion entre particules et substrat. On ne peut donc pas se limiter aux forces de Van der Walls pour décrire l'interaction particule-surface. Le rôle de cette humidité est difficile à quantifier car ses effets sont multiples :

- augmentation de l'adhésion par force de capillarité,

- modification des forces de Van der Walls par un effet d'écrantage,

- induire un mécanisme d'éjection par évaporation explosive du ménisque liquide,

- modification de la répartition d'énergie laser autour des particules.

Des expériences menées en chauffant le substrat devraient permettre de s'affranchir de cette humidité et de déterminer si l'évaporation explosive du ménisque présent sous les particules est bien à l'origine de leur éjection en régime d'irradiation à faible fluence.

\section{ENLÈVEMENT DE POLYMÈRES SUR WAFERS}

La finalité appliquée de ces travaux est d'évaluer la potentialité d'un procédé de nettoyage par laser dans le domaine de la microélectronique. Des essais ont donc été réalisés sur des wafers de silicium à 

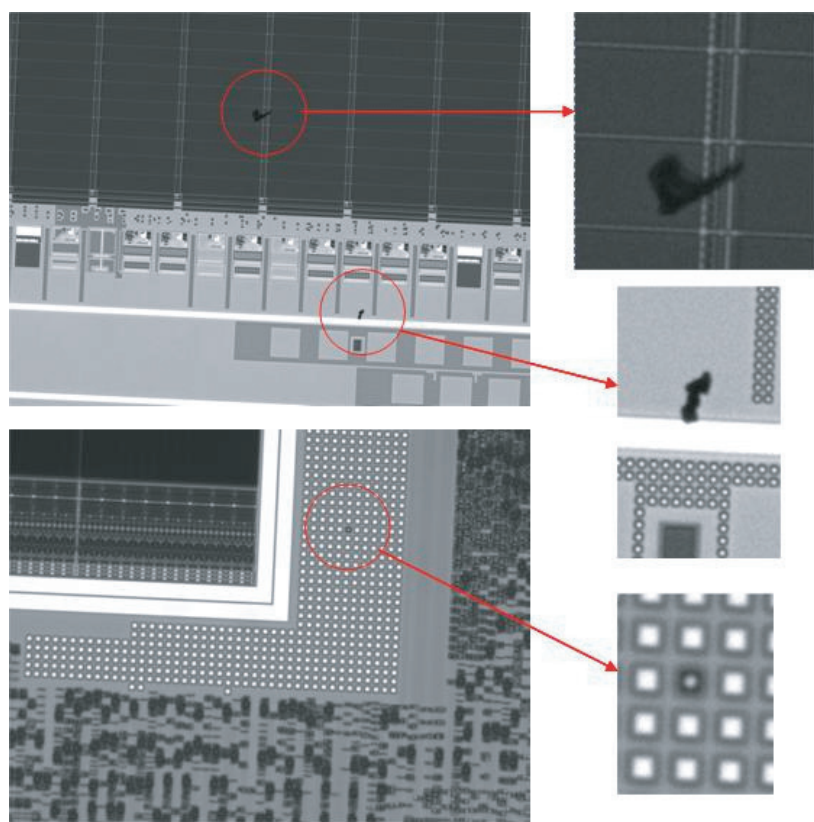

Avant
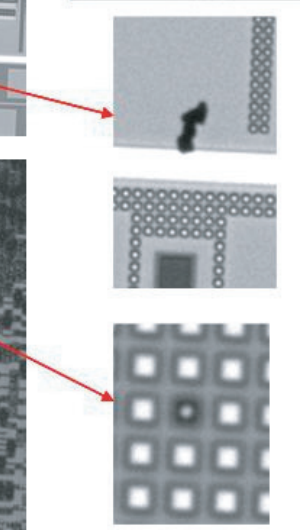

Zoom
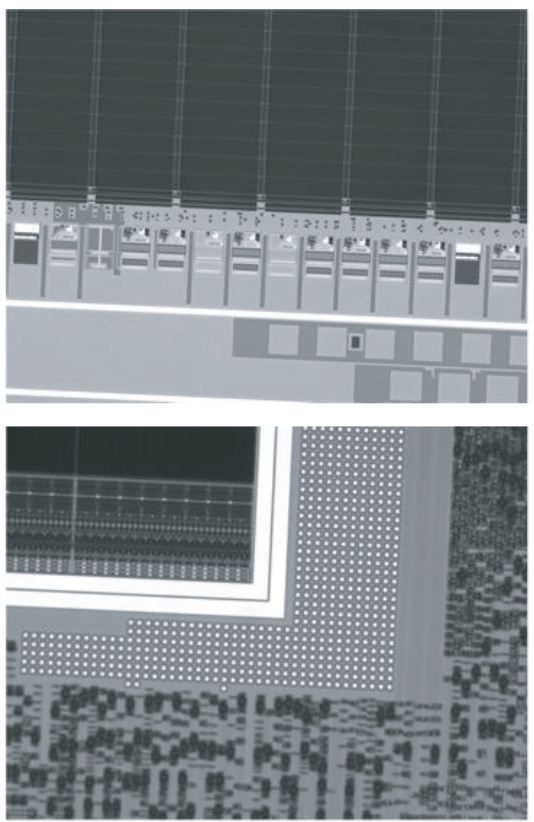

Après

Figure 12. Exemples d'enlèvement de polluants sur des wafers par irradiation laser.

différentes étapes de leur fabrication. Contrairement à l'étude des mécanismes présentée précédemment, les polluants présents à la surface des wafers n'étaient pas des composés transparents à la longueur d'onde du laser. L'ablation locale du substrat par exaltation en champ proche n'était donc pas à craindre. Les polluants étaient principalement des résidus de résine, qui étaient restés fixés à la surface malgré les différents procédés de nettoyage utilisés (scrubber, chimie). L'irradiation laser a été réalisée à $193 \mathrm{~nm}(\mathrm{ArF})$ dans une salle blanche de classe 1. Les polluants étant fortement absorbants à la longueur d'onde du laser ArF, ils sont rapidement ablatés, même pour des fluences faibles. Les particules les plus grosses, sont d'abord dissociées en éléments plus petits lors du premier tir, et disparaissent ensuite lors des tirs suivants. La figure 12 présente les résultats obtenus lors du nettoyage de wafers par laser. La fluence utilisée était inférieure à $100 \mathrm{~mJ} / \mathrm{cm}^{2}$, ce qui garantissait l'innocuité du procédé.

\section{CONCLUSION}

Cette étude sur l'enlèvement de particules par laser a démontré que, contrairement aux hypothèses de nombreuses études théoriques, l'expansion rapide de la surface due à son échauffement par le faisceau laser n'est pas le processus responsable de l'éjection. Les mesures de temps de vol du nuage de particules ont mis en évidence deux régimes de nettoyage. A forte fluence l'ablation locale du substrat induite par une surintensité due à l'exaltation en champ proche sous la particule est le mécanisme responsable de l'éjection. Pour les fluences plus proches du seuil de nettoyage, les mécanismes ne sont pas encore clairement identifiés, mais il semble que l'humidité résiduelle piégée entre la particule et le substrat joue un rôle important.

Les expériences de nettoyage par laser menées sur des wafers à différentes étapes de leur fabrication démontrent que ce procédé permet, dans certaines conditions, d'enlever des polluants pour lesquels les autres procédés se sont avérés inefficaces. 
Les applications potentielles de cette technique d'enlèvement de particules dans le domaine de l'ultra-propreté sont donc particulièrement attrayantes, et une compréhension plus complète des mécanismes d'éjection devrait encore permettre d'optimiser ce procédé.

\section{Remerciements}

Ces travaux ont bénéficié d'un soutien financier du Conseil Général des Bouches du Rhône, au travers du programme de recherche Rousset 2003-2008. Les auteurs remercient M. Laurent Charmasson (LP3) pour son efficacité et son ingéniosité dans la réalisation des dispositifs expérimentaux ainsi que messieurs Serge Nitsche et Damien Chaudansson (CRMC-N) pour leur aide lors des analyses par microscopie électronique.

\section{Références}

[1] B. S. Luk'yanchuk, Z. B. Wang, W. D. Song, and M. H. Hong, Appl. Phys. A 79, p. 747-751 (2004)

[2] D. Kane, A. Fernandes, D. Halfpenny, 'Laser Cleaning', World Scientific Publishing, B. Luk'yanchuk Editor, p. 181-228 (2002)

[3] W. Zapka, W. Ziemlich, A. Tam, Appl. Phys. Lett. 58 (20), p. 2217-2219 (1991)

[4] Y. Lee, Y. Lu, D. Chan, T. Low, M. Zhou, Jpn. J. Appl. Phys. 37, p. 2524-2529 (1998)

[5] M. Mosbacher, H.-J. Münzer, J. Zimmermann, J. Solis, J. Boneberg and P. Leiderer, Appl. Phys. A 72, p. 41-44 (2001)

[6] D. Grojo, M. Boyomo-Onana, A. Cros, Ph. Delaporte, Appl. Surf. Sci. 252 (13), p. 4786-4791 (2006)

[7] N. Arnold, 'Laser Cleaning', World Scientific Publishing, B. Luk'yanchuk Editor, p. 51-102 (2002)

[8] B. Luk'yanchuk, Y. Zheng, Y. Lu, Proc. SPIE 4423, p. 115-126 (2001)

[9] N. Arnold, Appl. Surf. Sci. 15, p. 208-209 (2003)

[10] Luk'yanchuk, M. Hong, Y. Lin, T. Chong, Phys. Rev. B 70, 035418 (2004)

[11] G. Vereecke, E. Röhr, and M. Heyns, J. Appl. Phys. 85, p. 3837 (1999)

[12] Y. Zheng, B. Luk'yanchuk, Y. Lu, W. Song, Z. Mai, J. Appl. Phys. 90, p. 2135-2142 (2001)

[13] V. Dobler, R. Oltra, J. P. Boquillon, M. Mosbacher, J. Boneberg, P. Leiderer, Appl. Phys. A 69, p. S335-S337 (1999)

[14] D. Grojo, A. Cros, Ph. Delaporte, M. Sentis, Appl. Phys. B, sous presse, (2006)

[15] B. Luk'yanchuk, N. Arnold, S. Huang, Z. Wang, M. Hong, Appl. Phys. A 77, p. 209-215 (2003)

[16] Luk'yanchuk, Z. Wang, L. Shi, T. Chong, Appl. Phys. Lett. 88, 023110 (2006)

[17] M. Mosbacher, M. Bertsch, H. Münzer, V. Dobler, B. Runge, D. Bäuerle, J. Boneberg, P. Leiderer, SPIE Proceedings 4426, p. 308 (2002)

[18] F. Lang, M. Mosbacher, P. Leiderer, Appl. Phys. A 77, p. 117-123 (2003)

[19] O. Yavas, A. Schilling, J. Bischof, J. Boneberg, P. Leiderer, Appl. Phys. A 64 (4), p. 331-339 (1997)

[20] W. Zapka, 'Laser Cleaning', B. Luk'yanchuk (Ed.), World Scientific Publishing, p. 23-48 (2002) 